\title{
BMJ Open Intubation performance using different laryngoscopes while wearing chemical protective equipment: a manikin study
}

\author{
H Schröder, ${ }^{1,2} \mathrm{~N}$ Zoremba, ${ }^{1,3} \mathrm{R}$ Rossaint, ${ }^{1} \mathrm{~K}$ Deusser, ${ }^{4} \mathrm{C}$ Stoppe, ${ }^{1} \mathrm{M}$ Coburn, ${ }^{1}$ \\ A Rieg, ${ }^{1}$ G Schälte ${ }^{1}$
}

To cite: Schröder $\mathrm{H}$,

Zoremba N, Rossaint R, et al. Intubation performance using different laryngoscopes while wearing chemical protective equipment: a manikin study. BMJ Open 2016;6:e010250. doi:10.1136/bmjopen-2015010250

- Prepublication history for this paper is available online. To view these files please visit the journal online (http://dx.doi.org/10.1136/ bmjopen-2015-010250).

Received 12 October 2015 Revised 8 January 2016 Accepted 14 January 2016

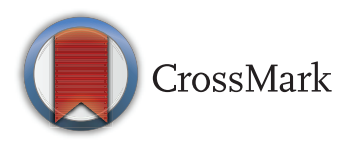

${ }^{1}$ Department of Anesthesiology, University Hospital RWTH Aachen, Aachen, Germany

${ }^{2}$ Department of Operative Intensive Care and Intermediate Care, University Hospital RWTH Aachen, Aachen, Germany

${ }^{3}$ Department of

Anesthesiology and Intensive Care, St Elisabeth Hospital, Gütersloh, Germany

${ }^{4}$ Department of Internal Medicine, Aachen District Medical Center, Würselen, Germany

Correspondence to Dr G Schälte;

gschaelte@ukaachen.de

\section{ABSTRACT}

Objectives: This study aimed to compare visualisation of the vocal cords and performance of intubation by anaesthetists using four different laryngoscopes while wearing full chemical protective equipment.

Setting: Medical simulation center of a university hospital, department of anaesthesiology.

Participants: 42 anaesthetists ( 15 females and 27 males) completed the trial. The participants were grouped according to their professional education as anaesthesiology residents with experience of $<2$ years or $<5$ years, or as anaesthesiology specialists with experience of $>5$ years.

Interventions: In a manikin scenario, participants performed endotracheal intubations with four different direct and indirect laryngoscopes (Macintosh (MAC), Airtraq (ATQ), Glidescope (GLS) and AP Advance $(A P A)$ ), while wearing chemical protective gear, including a body suit, rubber gloves, a fire helmet and breathing apparatus.

Primary and secondary outcome measures: With respect to the manikin, setting time to complete 'endotracheal intubation' was defined as primary end point. Glottis visualisation (according to the CormackLehane score (CLS) and impairments caused by the protective equipment, were defined as secondary outcome measures.

Results: The times to tracheal intubation were calculated using the MAC $(31.4 \mathrm{~s} ; 95 \% \mathrm{Cl} 26.6$ to 36.8), ATQ (37.1 s; $95 \%$ Cl 28.3 to 45.9 ), GLS (35.4 s; $95 \% \mathrm{Cl} 28.7$ to 42.1 ) and APA (23.6 s; $95 \% \mathrm{Cl} 19.1$ to 28.1), respectively. Intubation with the APA was significantly faster than with all the other devices examined among the total study population $(p<0.05)$. A significant improvement in visualisation of the vocal cords was reported for the APA compared with the GLS.

Conclusions: Despite the restrictions caused by the equipment, the anaesthetists intubated the manikin successfully within adequate time. The APA outperformed the other devices in the time to intubation, and it has been evaluated as an easily manageable device for anaesthetists with varying degrees of experience (low to high), providing good visualisation in scenarios that require the use of chemical protective equipment.

\section{Strengths and limitations of this study}

- To the best of our knowledge, video laryngoscopes have been neither sufficiently assessed nor compared under personnel using chemical protective equipment (CPE). This study aimed to compare three types of optical and video laryngoscopes with the standard Macintosh, examining their influences on the ease and speed of insertion of an endotracheal tube while wearing CPE.

- The AP Advance laryngoscope (video-extended standard laryngoscope) has been proven to be an easily manageable device for anaesthetists with varying experience levels (low to high) and has been demonstrated to allow for adequate visualisation in scenarios requiring the use of CPE. Nevertheless, the use of all devices tested is feasible and safe.

- A limitation of our study is the artificiality of difficult airway simulation using a manikin, which could have affected the scenario and had a training effect on the participants.

- The cohort of participants consisted of anaesthetists with different levels of experience. We did not include paramedics in this study. This aspect needs to be considered depending on the emergency medical service available.

- Further investigation into the efficacy and outcomes of securing the airway in real prehospital emergencies is required.

\section{INTRODUCTION}

Chemical, biological, radiation or nuclear (CBRN) hazards due to industrial activities, transport accidents, warfare incidents, communicable diseases or even terrorist attacks, can endanger the public. ${ }^{1}$ The uncontrolled release of toxic or contagious agents can lead to harmful inhalation and cause respiratory failure, which can require on-site treatment and the retaining of a secure airway. ${ }^{2} 3$ Immediate prehospital treatment, including early airway management, must be performed before decontamination or 
evacuation to avoid delayed intubation and the negative consequences on patients' outcomes. ${ }^{2}{ }^{4}$ Hazards, such as the Ebola outbreak in West Africa in 2014, confront hospital personnel with the need to intubate patients with respiratory failure using a video laryngoscope while wearing full personal protective equipment (PPE). ${ }^{5}$

The wearing of CBRN-PPE gear, including rubber gloves and a fire helmet, visor and hood, results in impaired manual dexterity and limited vision during intubation. ${ }^{6}$ This impairment adversely affects the successful performance of endotracheal intubation, ${ }^{7}$ as well as the use of oropharyngeal airway devices. ${ }^{8}$ Furthermore, the positions of patients, who are most likely to be lying on the floor, can result in prolonged intubation times and increase the number of failed intubation attempts. ${ }^{9}$

Videolaryngoscopy may be beneficial for performance of successful endotracheal intubation under difficult conditions, due to their improved ability to provide adequate glottic visualisation. ${ }^{10}{ }^{11}$ However, there is little current evidence for or against the use of videolaryngoscopy when wearing chemical protective equipment (CPE). ${ }^{12} 13$ The aim of the current small, unblinded, pilot study is to describe the performance characteristics of various laryngoscopic techniques.

This study compares three types of laryngoscopes with the standard Macintosh (MAC) laryngoscope, examining their influences on the ease and speed of insertion of an endotracheal tube by operators wearing CBRN-PPE. We further assessed the operators' subjective impressions of the devices, with a focus on the ability to adequately visualise the glottis.

\section{METHODS}

\section{Participants}

Forty-two anaesthetists from Aachen University Hospital, Germany, were invited to participate in this comparative pilot study. The ethical committee waived the need to obtain written informed consent. All of the participants agreed to have their performances evaluated and anonymously used for scientific and educational purposes. The prerequisite for inclusion was an educational level of at least a first year residency in anaesthesiology, implying the possession of a license to practise medicine.

\section{Equipment}

A Laerdal Resusci Anne, including an Anne Airway Trainer Update Kit (Laerdal Medical GmbH, Puchstein, Germany), was chosen as the manikin type for this study, and $7 \mathrm{~mm}$ endotracheal cuffed tubes were used for intubation (best fit). The manikin's airway was lubricated with silicon spray before and cleaned after each insertion.

All of the tasks were completed while the participants were wearing ISOTEMP-4000 chemical protection gear, including a complete body suit, rubber gloves and German DIN 14940 fire helmets with a $15 \mathrm{~kg}$ self-contained breathing apparatus beneath (Dräger AG, Lübeck, Germany). Since the use of the breathing apparatus requires special training and qualification, the participants did not connect it.

For comparison, the following four laryngoscopic intubation devices were selected: a MAC (a conventional standard laryngoscope used worldwide), an Airtraq A-011 (ATQ) (an established single-use indirect laryngoscope offering optics and a guiding channel) (Prodol Ltd, Vizcaya, Spain), a Glidescope (GLS) (a video laryngoscope that allows for high-quality visualisation on an external monitor, with no guiding channel) (Verathon Medical BV, Rennerod, Germany) and an AP Advance (APA) (video extended standard laryngoscope with a directly attached display and certain blade options, including a difficult airway blade (DAB), with a guiding channel) (Venner Medical GmbH, Dänischenhagen, Germany) (figure 1).

The device sizes and blades were found to be congruent and were fit to the manikin's specifics prior to testing. All of the devices were used with blades that were equivalent to a size $3 \mathrm{MAC}$ blade and, for the APA specifically, a DAB. A standard intubation stylet (14 Charrière $(\mathrm{CH})$ ) was applied for use with the conventional MAC. For the GLS, a GlideRite stylet was used for intubation according to the manufacturer's recommendations. No stylets were used for the ATQ and APA because these devices offer a guiding channel.

\section{Study protocol}

Each participant was familiar with all of the devices tested, based on the receipt of previous 'managing the difficult airway' education and subsequent manikin training. Before testing, all participants were once again instructed on the correct techniques for using the laryngoscopes. The participants were not allowed to practise any tasks in their chemical protection gear. Two participants declared that they had former training experience with chemical protective gear.

In the scenario, the manikin was placed on the floor with all four devices preassembled and easily accessible, close to the manikin's head (figure 1). Each participant performed the complete process of intubation with each device, from grasping of the device until the first ventilation with a bag-valve mask. The process included inflation of the cuff.

Successful intubation was identified as regular chest extension of the manikin and was further verified by the authors after completion. The order of devices 1 to 4 (MAC, ATQ GLS and APA, respectively) was rotated for every second participant, from the sequence $1,2,3,4$ to $2,3,4,1$ to $3,4,1,2$ and finally to $4,1,2,3$, to compensate for potential learning bias.

\section{Data assessment}

The anaesthetists were assessed by the study team, and the time to complete intubation was recorded from the entrance of each laryngoscope through the mouth until 
Figure 1 Devices and equipment: upper left Airtraq (ATQ); upper right Glidescope (GLS); lower left ISOTEMP Chemical Protection Equipment (CPE); lower right AP Advance (APA).

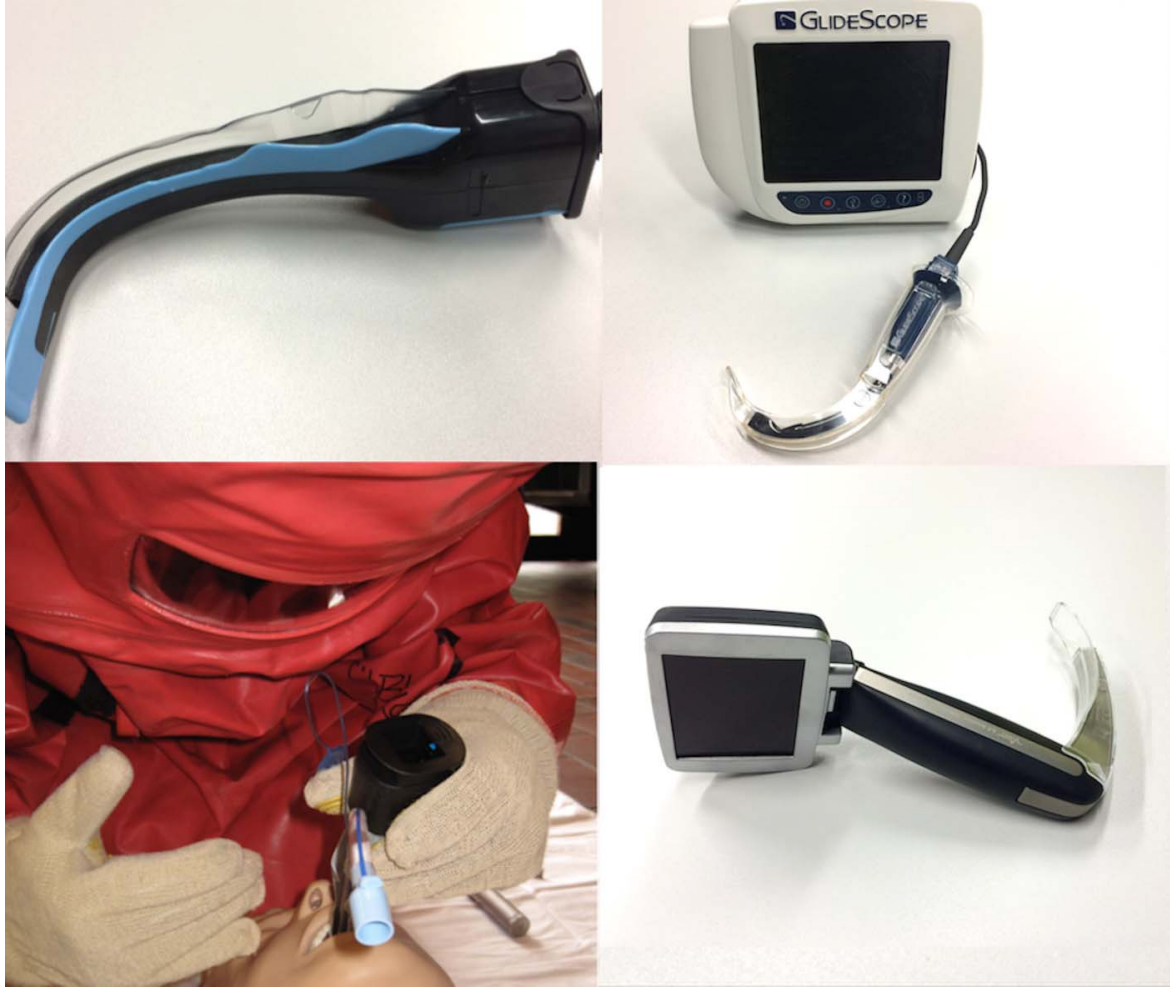

the moment of chest extension by the first ventilation. After completion of all four intubations, the participants were administered a questionnaire on the difficulties experienced during the performance. The questionnaire included restrictions in handling the devices caused by the gear, as well general comments about their handling, rated on a numeric scale from 1 (no restriction) to 7 (maximum restriction). To qualify the visualisation of the vocal cords, we used the standard Cormack-Lehane (CL) classifications of I-IV for classic direct laryngoscopy. To achieve comparability between direct, indirect and video laryngoscopy in the absence of an alternative practical score, we chose to use the CL classification to assess visualisation with all devices, although it is typically used only for direct laryngoscopy. The data were collected over 11 days, with an average daily assessment of 4 participants.

\section{Statistical analysis}

The results are presented as the mean $\pm \mathrm{SD}(\mathrm{M} \pm \mathrm{SD})$ for the continuous variables. The parameters were compared using the Friedman test as an alternative to analysis of variance ANOVA for non-parametric groups. Bonferroni-Dunn correction was used to determine the significance of data. Comparisons were considered statistically significant at a $\mathrm{p}<0.05$. Statistical analysis was conducted with Prism 5 software (V.5.0 for Mac OS X, copyright 1994-2009, GraphPad).

\section{RESULTS}

Data were recorded for 42 anaesthetists (15 females and 27 males). The participants were grouped according to their professional education as anaesthesiology residents with experience of $<2$ years or $<5$ years or as anaesthesiology specialists with experience of $>5$ years (table 1 ).

Wearing CBRN-PPE, all of the participants successfully intubated the manikin's trachea with the MAC, GLS and APA. One inaccurate intubation was recorded for the ATQ. The time to tracheal intubation using the MAC was $31.7 \pm 16.3 \mathrm{~s}($ mean \pm SD) (range 13.8-96.4), and it was $37.1 \pm 28.2 \mathrm{~s}(12.1-156.0)$ using the ATQ, 35.4 $\pm 21.6 \mathrm{~s}$ (13.5-93.3) using the GLS and 23.6 $\pm 14.5 \mathrm{~s}$ (11.4-99.4) using the APA. Intubation using the APA was significantly faster compared with all of the other devices among the total study population (table 2; figure 2).

Regarding the level of experience, the residents with $<2$ years experience performed intubation significantly faster $(\mathrm{p}<0.05)$ with the APA compared with the GLS $(23.7 \pm 8.6 \mathrm{~s}$ vs $46.6 \pm 25.7 \mathrm{~s}$, respectively). The specialists with $>5$ years experience were significantly faster with the APA compared with the GLS and MAC $(21.9 \pm 18.5 \mathrm{~s}$ vs $32.4 \pm 21.8 \mathrm{~s}$ and $28.5 \pm 14.7 \mathrm{~s}$, respectively; table 2 ).

The participants completed the questionnaire by subjectively assessing the restrictions of visualisation and laryngoscope handling caused by the protective gear

\begin{tabular}{lccc} 
Table 1 & Participant characteristics & & \\
\hline & Participants & Male & Female \\
\hline Total & 42 & 27 & 15 \\
Residents 0-2 years & 10 & 4 & 6 \\
Residents 2-5 years & 12 & 7 & 5 \\
Specialists & 20 & 16 & 4 \\
\hline Data are presented as numbers. & &
\end{tabular}


Table 2 Differences in the time for intubation according to the device used and level of professional experience

\begin{tabular}{lllll}
\hline Total participants & MAC & ATQ & GLS & APA \\
\hline Successful intubation & 42 & 41 & 42 & 42 \\
Minimum & 13.8 & 12.1 & 13.5 & 11.4 \\
Maximum & 96.4 & 156 & 93.3 & 99.4 \\
Mean & 31.4 & 37.1 & 35.4 & $23.6^{\star}$ \\
SD & 16.3 & 28.2 & 21.6 & 14.5 \\
Residents <2 years & & & & \\
Successful intubation & 10 & 10 & 10 & 10 \\
Minimum & 16.3 & 17.3 & 20 & 14.2 \\
Maximum & 63.3 & 59.2 & 92.2 & 38.75 \\
Mean & 34.9 & 34.3 & 46.6 & $23.7 \dagger$ \\
SD & 13.8 & 13.7 & 25.7 & 8.6 \\
Residents 2-5 years & & & & \\
Successful intubation & 12 & 12 & 12 & 12 \\
Minimum & 16.8 & 22.3 & 17.9 & 17.9 \\
Maximum & 96.4 & 156 & 66.6 & 49.5 \\
Mean & 33.2 & 47.8 & 31.0 & 26.4 \\
SD & 20.8 & 39.1 & 14.7 & 10.9 \\
Specialists >5 years & & & & \\
Successful intubation & 20 & 19 & 20 & 20 \\
Minimum & 13.8 & 12.1 & 13.45 & 11.4 \\
Maximum & 78.1 & 99.3 & 93.3 & 99.4 \\
Mean & 28.5 & 31.9 & 32.4 & $21.9 \ddagger$ \\
SD & 14.7 & 25.1 & 21.8 & 18.5 \\
\hline Da are presented & & &
\end{tabular}

Data are presented as numbers, time(s) and means \pm SDs. ${ }^{*}$ Tracheal intubation was significantly faster using the APA compared with all of the other devices $(p<0.05)$.

tResidents with experience of $<2$ years performed intubation significantly faster with the APA than with the GLS $(p<0.05)$. $\ddagger$ Specialists with experience of $>5$ years performed intubation significantly faster with the APA than with the GLS or MAC $(p<0.05)$.

APA, AP Advance; ATQ, Airtraq; GLS, Glidescope; MAC, Macintosh. (figure 3) using a numeric scale ranging from 1 (no restriction) to 7 (maximum restriction). The questionnaire results showed that $45 \%$ of the participants achieved CL-I visualisation with the MAC, in addition to $62 \%$ with the ATQ $88 \%$ with the GLS and $95 \%$ with the APA. A significant difference in the rank sum was detected between the APA (42.9) and GLS (37.5) $(\mathrm{p}<0.05$; figure 4$)$.

Evaluation of restriction of visualisation caused by the CBRN-PPE resulted in the highest score (indicating the greatest restriction) for the MAC (4.3), followed by the ATQ (4.2) and the GLS (2.7), and the lowest score was observed for the APA (2.4). Wearing PPE restricted the handling of the MAC, ATQ, GLS and APA laryngoscopes with associated scores of 3.8, 4.1, 3.0 and 2.6, respectively. In addition, the participants compared their former experiences with using these laryngoscopes without wearing CBRN-PPE with their current experiences, and scored the restriction caused by the PPE as 4.9 for the MAC, 4.5 for the ATQ 3.3 for the GLS and 2.8 for the APA (figure 3 ).

Within the free comment section of the questionnaire, the participants indicated that they had experienced

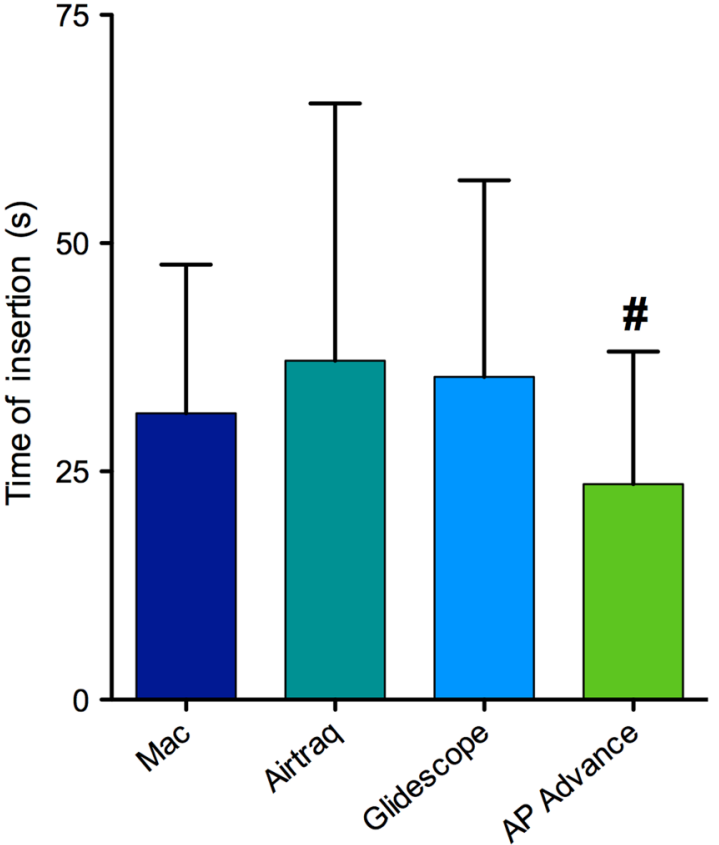

Figure 2 Intubation times for all devices.

major problems in adjusting the angle of view (41\%) for the ATQ. In addition, $45 \%$ of the participants reported major difficulty with obtaining a sufficient glottic view using the MAC. Impairments of manual dexterity and fine motor skills were confirmed by 36 participants $(86 \%)$, specifically for advancing the tube into the trachea $(36 \%)$. In response to the question regarding which laryngoscope they would prefer to use while wearing CBRN-PPE, 4 chose the MAC, 1 chose the ATQ 16 selected the GLS and 18 opted for the APA. Three participants did not answer this question.

\section{DISCUSSION}

In this trial, we have demonstrated that video laryngoscopes are feasible, safe and easy to handle-even when wearing CBRN-PPE-for use on a manikin. Compared to conventional MAC laryngoscopy, the video laryngoscopes allowed for better visualisation during intubation, despite the fact that the participants were looking through a glass shield integrated into the protective gear. Moreover, the APA outperformed the other laryngoscopes in terms of visualisation and the time to tracheal intubation.

Tracheal intubation in prehospital situations should always be performed by the most experienced medical staff. Since chemical intoxication and environmental circumstances can complicate intubation, it must be assessed whether a supraglottic airway device (SAD) can secure the airway sufficiently until an experienced physician and equipment are available.

SADs (eg, a laryngeal mask) have been evaluated for use with CBRN-CPE, and their benefits include ease of use and speed of insertion. Additionally, less stringent training requirements for their use have been 
Figure 3 Questionnaire data: restriction caused by chemical protective equipment. APA, AP Advance; ATQ, Airtraq; GLS, Glidescope; MAC, Macintosh.

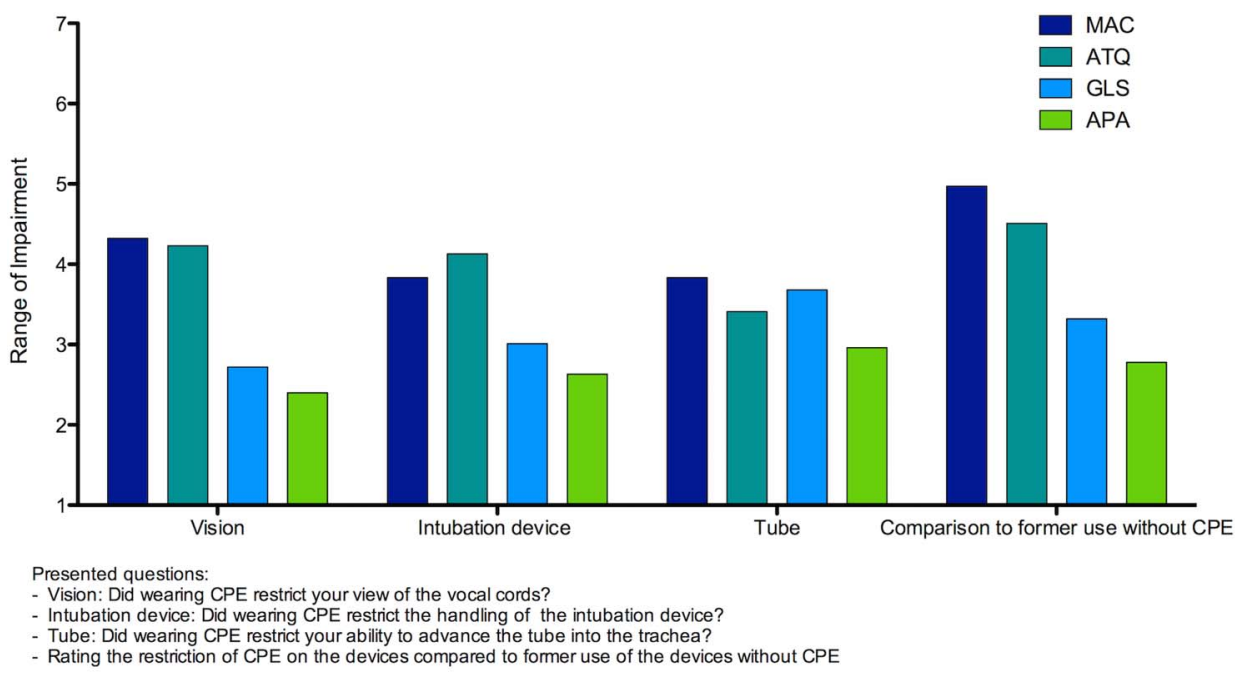

confirmed. ${ }^{14-16}$ Despite their advantages, SADs do not offer the same quality of separation of the respiratory and digestive tracts. ${ }^{17}$ Further, they do not provide adequate airway protection, particularly during positive pressure ventilation after respiratory intoxication. ${ }^{3}$ Thus, endotracheal intubation remains the gold standard in the early airway management of contaminated patients, to avoid the higher mortality rate resulting from the hypoxia caused by delayed intubation. ${ }^{2}$

Video laryngoscopy provides a better view of the vocal cords, a higher success rate, a shorter time to tracheal intubation and less need for optimising manoeuvres. $^{18}$ These devices are considered easy to use regardless of previous experience, and they have been recommended for difficult airway situations. ${ }^{18}{ }^{19}$ In prehospital settings, the option to perform both direct and video laryngoscopy with the same device has been emphasised. ${ }^{20}$

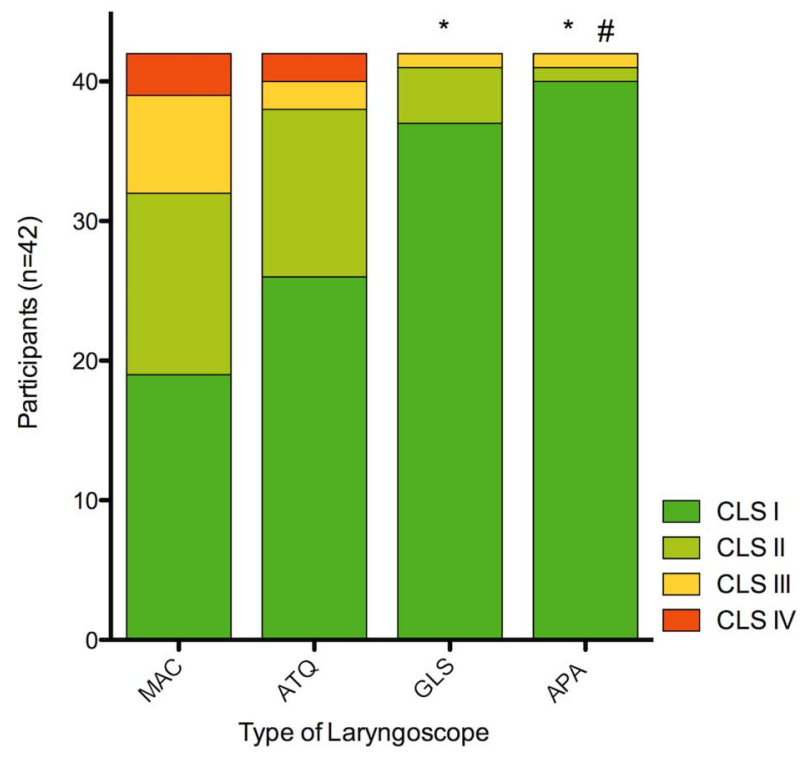

Figure 4 Visualisation according to intubation scores.
A short learning curve enables personnel to perform successful tracheal intubations with limited instruction. ${ }^{11}$ A previous study of the use of a Pentax-AWS video laryngoscope in a CBRN-PPE scenario has shown that suited intubation with a Pentax-AWS can be performed in a shorter period of time than unsuited intubation with an MAC. ${ }^{12}$

\section{Intubation performances with the different laryngoscopes}

Insertion of a tube through the glottis into the trachea is essential for performance analysis of the ease and speed of endotracheal intubation. This success of this manoeuvre is mainly influenced by the view of the vocal cords, and it certainly depends on the type of laryngoscope used for intubation. Both, the presence or absence of a guiding channel on a laryngoscope and the relevant intubation technique, requiring visual and manual coordination, can influence the outcome. Although most video laryngoscopes provide high-quality visualisation of the anatomy, successful tracheal intubation is not guaranteed.

The APA performed the best in this trial with regard to speed and ease of intubation. The monitor of the APA is directly aligned to the laryngoscope with its familiar shape, enabling anaesthetists to apply well-known intubation techniques, similar to the standard MAC. Additionally, the APA offers high-quality visualisation, and the guiding channel of the blade directs the path for the tube into the trachea. These advantages of the APA can be transferred from a standard airway scenario $^{21}$ to our scenario involving the wearing of complete CBRN-PPE.

The mean intubation time with the GLS was comparable to that with the ATQ and both of these laryngoscopes required more time than the standard MAC. Anaesthetists with less than 2 years of experience performed intubation more slowly with the GLS than with the MAC or ATQ. Despite the excellent view quality on the external monitor, the visual and manual coordination required by the unfamiliar technique posed a 
challenge to the less experienced participants. Although the survey revealed that vision was less restricted with the GLS (compared to the MAC and ATQ), it further indicated limitations of the GLS in advancing the tube into the trachea. Similar findings for the GLS have been demonstrated under non-CPE-wearing conditions. ${ }^{21}$

In particular, the GLS requires even greater dexterity under PPE-wearing conditions, and the participants' performances would have benefitted from a training period to familiarise them with the coordination necessary for successful and fast intubation with this laryngoscope. The use of the ATQ with indirect laryngoscopy optics and a clear guiding channel for the tube, has been investigated as a feasible device for performing routine tracheal intubation rapidly. ${ }^{22}$ However, in our scenario, involving the wearing of CBRN-PPE, this laryngoscope did not demonstrate such efficiency. In fact, its use resulted in the slowest intubation times.

The fixed angle of the ATQ might have limited the possible adjustments made by the operators for achieving adequate visualisation of the vocal cords while they were protected by the visor of the helmet and suit, which would have additionally restricted the use of the eyepiece of the optical unit. These limitations were mentioned in the survey by more than $40 \%$ of the participants. Castle et al observed the longest intubation time for participants using the ATQ while wearing CBRN-PPE. However, in contrast with our findings, their study did not describe interference from a helmet or visor. ${ }^{14} 15$ Recently, Claret et $a l^{23}$ confirmed that the MAC is superior to the ATQ in terms of speed, efficiency and overall ease of use.

The MAC laryngoscope is a well-known reference device with confirmed utility under difficult circumstances. Although the intubation times for the MAC were adequate and were only exceeded by those for the APA in this study, the anaesthetists' experienced restrictions of visualisation and laryngoscope handling while wearing CBRN-PPE, as demonstrated by the CL classification and questionnaire results. Notably, the anaesthetists' greater experience with using the MAC compensated for the lack of visualisation compared with the other devices.

\section{Survey: restrictions from CBRN-PPE}

The participants confirmed that the protective gear, including rubber gloves and a fire helmet, visor and hood, impaired the performance of endotracheal intubation. ${ }^{6} 7$ Fine motor skills were needed for advancing the tube into the trachea and for inflation of the cuff, and the participants claimed to be restricted mainly due to the rigidity and inflexibility of the rubber gloves.

With regard to visualisation of the glottis, the participants indicated that they were less restricted by the PPE gear when using the APA video laryngoscope (figure 3). The high quality of the resulting image may explain this finding, as well as the fact that the display of the APA is directly attached to the familiar body of the laryngoscope. Visualisation of the vocal cords does not require averting one's eyes to a separate monitor, similar to the GLS. To acquire an adequate view with the MAC, the participants had to align the glottis, laryngoscope and themselves, but they were specifically restricted by the visor and hood of the protective gear, and the position of the manikin on the floor, as mentioned by 19 of the 42 participants. Castle $e t a l^{14}{ }^{15}$ have shown that performing intubation with the ATQ is not limited by the distance between the eyepiece and laryngoscope, which is increased by the visor of the gear. In contrast, 17 of the 42 participants in our study specifically reported this distance as the reason for an impaired view while intubating with the ATQ.

Our findings were further supported by the recording of CLSs for the use of indirect laryngoscopy and video laryngoscopy. It has been shown that the best visualisation is achieved using the APA, followed by the GLS, ATQ and MAC. We also found that the quality of visualisation was not correlated with the time of intubation, because the MAC outperformed the GLS and ATQ despite the poorer visualisation.

\section{Limitations}

We recognise that the artificial scenario for difficult airway assessment was limited by the lack of proper simulation of collapsible soft tissues and secretion of blood, vomit or sputum, as well as the use of rigid plastic, which established the scenario and could have had a training effect on the participants. ${ }^{24}$ Additionally, chemical incidents occurring in or out of hospital can be further assessed according to the presence of fog, fire, noise, dirt or other external conditions. Considering the setting and conditions of our study, with the participants wearing CPE and the manikin lying on the floor, we defined this as a difficult airway scenario without considering the standard anatomic glottic location of the manikin. The American Society of Anesthesiologists (ASA) Task Force on Management of the Difficult Airway has defined the difficult airway as a complex interaction among patient factors, the clinical setting and the skills of the practitioner. ${ }^{25}$

Particularly with the use of the APA, which consists of a plastic guiding channel on its downside, the friction of rigid plastic on the manikin's plastic larynx might have worsened the outcome. Furthermore, the DAB of the APA might have facilitated the use of the device, while the MAC and GLS options were restricted to the use of a stylet. In addition, the ATQ has a guiding channel for support. Moreover, we did not consider the blinding of the observers to the tested devices, as it was not possible to acquire a 'neutral' image quality that would prevent the experienced observer from determining the device used. We also did not consider aspects such as battery life span or economic feasibility in our study. Owing to the lack of a reliable quantitative parameter for the primary goal of success, we chose 'time to successful intubation' as our best descriptive term. 


\section{Conclusions}

The APA has demonstrated to be an easily manageable device for anaesthetists with various experience levels (low to high); and it can be used to achieve adequate visualisation in scenarios requiring CPE. Emergency scenarios require devices that are not only easy to use but are also well known by staff. Therefore, the MAC remains a reliable option for anaesthetists in the field, even under complicated conditions. The findings of this study serve as a foundation for further clinical studies on outcomes of securing the airway using video laryngoscopes, such as the APA.

Contributors HS and NZ equally developed the conception and design of the study, performed the data interpretation, and drafted and finalised the manuscript. GS and KD participated in the data collection and interpretation. CS, MC and RR critically revised the manuscript and supervised statistical analysis. RR and GS critically revised the manuscript, and GS initiated, coordinated and supervised the trial. All of the authors read and approved the final manuscript.

Funding This research received no specific grant from any funding agency in the public, commercial or not-for-profit sectors.

Competing interests GS received fees for general lectures in 'difficult airway management' from the German Airtraq distributor Medisize Deutschland $\mathrm{GmbH}$, Neunkirchen-Seelscheid, Germany.

Ethics approval Rhine-Westphalia University of Technology Aachen, Medical Faculty, Ethical Review Committee, approval number EK 115/12.

Provenance and peer review Not commissioned; externally peer reviewed.

Data sharing statement No additional data are available.

Open Access This is an Open Access article distributed in accordance with the Creative Commons Attribution Non Commercial (CC BY-NC 4.0) license, which permits others to distribute, remix, adapt, build upon this work noncommercially, and license their derivative works on different terms, provided the original work is properly cited and the use is non-commercial. See: http:// creativecommons.org/licenses/by-nc/4.0/

\section{REFERENCES}

1. Murray V, Goodfellow F. Mass casualty chemical incidents-towards guidance for public health management. Public Health 2002;116:2-14.

2. Ben-Abraham R, Rudick V, Weinbroum AA. Practical guidelines for acute care of victims of bioterrorism: conventional injuries and concomitant nerve agent intoxication. Anaesthesiology 2002;97:989-1004.

3. Rodgers GC, Condurache CT. Antidotes and treatments for chemical warfare/terrorism agents: an evidence-based review. Clin Pharmacol Ther 2010;88:318-27.

4. Baker DJ. Advanced life support for acute toxic injury (TOXALS). Eur J Emerg Med 1996;3:256-62.

5. West TE, Saint André-von Arnim A. Clinical presentation and management of severe Ebola virus disease. Ann Am Thorac Soc 2014:11:1341-50.

6. Coates MJ, Jundi AS, James MR. Chemical protective clothing; a study into the ability of staff to perform lifesaving procedures. $J$ Accid Emerg Med 2000;17:115-18.
7. Flaishon R, Sotman A, Ben-Abraham R, et al. Antichemical protective gear prolongs time to successful airway management: a randomized, crossover study in humans. Anaesthesiology 2004;100:260-6.

8. Ben-Abraham R, Flaishon R, Stoman A, et al. Cuffed oropharyngeal airway (COPA) placement is delayed by wearing antichemical protective gear. Emerg Med J 2008;25:847-50.

9. Castle N, Owen R, Clarke S, et al. Does position of the patient adversely affect successful intubation whilst wearing CBRN-PPE? Resuscitation 2010;81:1166-71.

10. McElwain J, Malik MA, Harte BH, et al. Comparison of the C-MAC videolaryngoscope with the Macintosh, Glidescope, and Airtraq laryngoscopes in easy and difficult laryngoscopy scenarios in manikins. Anaesthesia 2010;65:483-9.

11. Malik MA, O'Donoghue C, Carney J, et al. Comparison of the Glidescope, the Pentax AWS, and the Truview EVO2 with the Macintosh laryngoscope in experienced anaesthetists: a manikin study. Br J Anaesth 2009;102:128-34.

12. Shin DH, Choi PC, Na JU, et al. Utility of the Pentax-AWS in performing tracheal intubation while wearing chemical, biological, radiation and nuclear personal protective equipment: a randomised crossover trial using a manikin. Emerg Med J 2013;30:527-31.

13. Piepho T, Weinert K, Heid FM, et al. Comparison of the Mcgrath $\circledast$ Series 5 and Glidescope $\circledR$ Ranger with the Macintosh laryngoscope by paramedics. Scand J Trauma Resusc Emerg Med 2011;19:4.

14. Castle N, Pillay Y, Spencer N. Insertion of six different supraglottic airway devices whilst wearing chemical, biological, radiation, nuclear-personal protective equipment: a manikin study. Anaesthesia 2011;66:983-8.

15. Castle N, Pillay Y, Spencer N. Comparison of six different intubation aids for use while wearing CBRN-PPE: a manikin study. Resuscitation 2011:82:1548-52.

16. Goldik Z, Bornstein J, Eden A, et al. Airway management by physicians wearing anti-chemical warfare gear: comparison between laryngeal mask airway and endotracheal intubation. Eur $J$ Anaesthesiol 2002;19:166-9.

17. Stone BJ, Chantler PJ, Baskett PJ. The incidence of regurgitation during cardiopulmonary resuscitation: a comparison between the bag valve mask and a laryngeal mask airway. Resuscitation 1998:38:3-6.

18. Jungbauer A, Schumann M, Brunkhorst V, et al. Expected difficult tracheal intubation: a prospective comparison of direct laryngoscopy and video laryngoscopy in 200 patients. Br J Anaesth 2009;102:546-50.

19. Powell L, Andrzejowski J, Taylor R, et al. Comparison of the performance of four laryngoscopes in a high-fidelity simulator using normal and difficult airway. Br J Anaesth 2009;103:755-60.

20. Cavus E, Callies A, Doerges V, et al. The C-MAC videolaryngoscope for prehospital emergency intubation: a prospective, multicentre, observational study. Emerg Med $J$ 2011;28:650-3.

21. Hodd JA, Doyle DJ, Gupta S, et al. A Mannequin study of intubation with the AP advance and GlideScope Ranger videolaryngoscopes and the Macintosh laryngoscope. Anesth Analg 2011;113:791-800.

22. Schälte G, Scheid U, Rex S, et al. The use of the Airtraq ${ }^{\circledR}$ optical laryngoscope for routine tracheal intubation in high-risk cardio-surgical patients. BMC Res Notes 2011;4:425.

23. Claret PG, Bobbia X, Asencio R, et al. Comparison of the Airtraq laryngoscope versus the conventional Macintosh laryngoscope while wearing CBRN-PPE. Eur J Emerg Med 2016;23:119-23.

24. Nakstad AR, Sandberg M. Airway management in simulated restricted access to a patient—can manikin-based studies provide relevant data? Scand J Trauma Resusc Emerg Med 2011;19:36.

25. Apfelbaum JL, Hagberg CA, Caplan RA, et al., American Society of Anesthesiologists Task Force on Management of the Difficult Airway Practice guidelines for management of the difficult airway: an updated report by the American Society of Anesthesiologists Task Force on Management of the Difficult Airway. Anesthesiology 2013;118:251-70. 


\section{Correction}

Schröder H, Zoremba N, Rossaint R, et al. Intubation performance using different laryngoscopes while wearing chemical protective equipment: a manikin study. BMJ Open 2016;6:e010250. doi:10.1136/bmjopen-2015-010250

There is a mistake in the Contributors section. It should say:

"Contributors HS and NZ developed the conception and design of the study and performed data interpretation. HS performed statistical analysis, wrote and finalized the manuscript. NZ revised the manuscript. GS and KD participated in the data collection and interpretation. CS, MC, RR critically revised the manuscript and supervised statistical analysis. RR and GS critically revised the manuscript, and GS initiated coordinated and supervised the trial. All of the authors read and approved the final manuscript".

Open Access This is an Open Access article distributed in accordance with the Creative Commons Attribution Non Commercial (CC BY-NC 4.0) license, which permits others to distribute, remix, adapt, build upon this work noncommercially, and license their derivative works on different terms, provided the original work is properly cited and the use is non-commercial. See: http://creativecommons.org/licenses/by-nc/4.0/

BMJ Open 2017;7:e010250corr1. doi:10.1136/bmjopen-2015-010250corr1 